\title{
Arrhythmias Associated with Inflammatory Cardiomyopathies
}

Roshan Karki, MBBS॰

Chaitra Janga, MBBS

Abhishek J. Deshmukh, MBBS ${ }^{*}$

\section{Address}

"Division of Cardiovascular Disease, Mayo Clinic, 200 1st Street, Rochester, MN, 55905, USA

Email: Deshmukh.Abhishek@mayo.edu

Published online: 19 November 2020

(C) Springer Science+Business Media, LLC, part of Springer Nature 2020

This article is part of the Topical Collection on Arrhythmia

Keywords Inflammatory cardiomyopathy · Arrhythmias · Cardiac sarcoidosis

Abbreviations AIC Arrhythmogenic inflammatory cardiomyopathy · CS Cardiac sarcoidosis GCM Giant-cell myocarditis

\section{Abstract}

Purpose of review To provide an approach to the diagnosis and treatment of arrhythmias associated with inflammatory cardiomyopathies.

Recent findings Inflammatory cardiomyopathies are increasingly recognized as the etiology of both ventricular and supraventricular arrhythmias. There have been recent studies providing novel insights into the pathogenesis of arrhythmias in inflammatory cardiomyopathies and exploring the role of various diagnostic tools and treatment strategies.

Summary Patients with inflammatory cardiomyopathies often present with one or more arrhythmias, including atrioventricular block, atrial and ventricular tachyarrhythmias, and occasionally sudden cardiac death. Given dynamic pathophysiology and heterogeneous presentation, the management of arrhythmias in these patients presents unique challenges. We review the current approach to the diagnosis and treatment of arrhythmias in this challenging cohort of patients with an emphasis on cardiac sarcoidosis. 


\section{Introduction}

Inflammatory cardiomyopathies (ICMs) are a spectrum of disorders characterized by acute or chronic inflammatory processes affecting the myocardium (myocarditis) and associated with mechanical cardiac dysfunction with altered hemodynamics, conduction disturbances, or electrical instability. Inflammatory cardiomyopathies are mostly caused by an infection or autoimmune disease that has been summarized in Table 1. The patients with ICMs frequently present with arrhythmias.
However, a subset of these patients may present first with arrhythmias and even sudden cardiac death, and are later diagnosed as ICMs [1]. Bauer and colleagues $[2 \bullet \bullet]$ proposed an operational diagnosis of "arrhythmogenic inflammatory cardiomyopathy" for these patients until a specific etiologic diagnosis is made. In the current review, we discuss an approach to the diagnosis and treatment of arrhythmias associated with ICMs with an emphasis on cardiac sarcoidosis (CS).

\section{Epidemiology}

Although the global annual incidence of reported myocarditis is 22 per 100,000 [3], the actual burden of arrhythmias associated with ICM is unknown. In an autopsy study ( $n=753$, age $<50$ years) of sudden cardiac deaths (SCDs), the prevalence of myocarditis was $6 \%$ [4]. Tung et al. demonstrated a $49 \%$ prevalence of occult myocardial inflammation by fluorodeoxyglucose positron emission tomography (FDG-PET) in selected patients referred for ventricular

Table 1. Inflammatory Cardiomyopathies cardiomyopathies and Associated associated Arrhythmiasarrhythmias

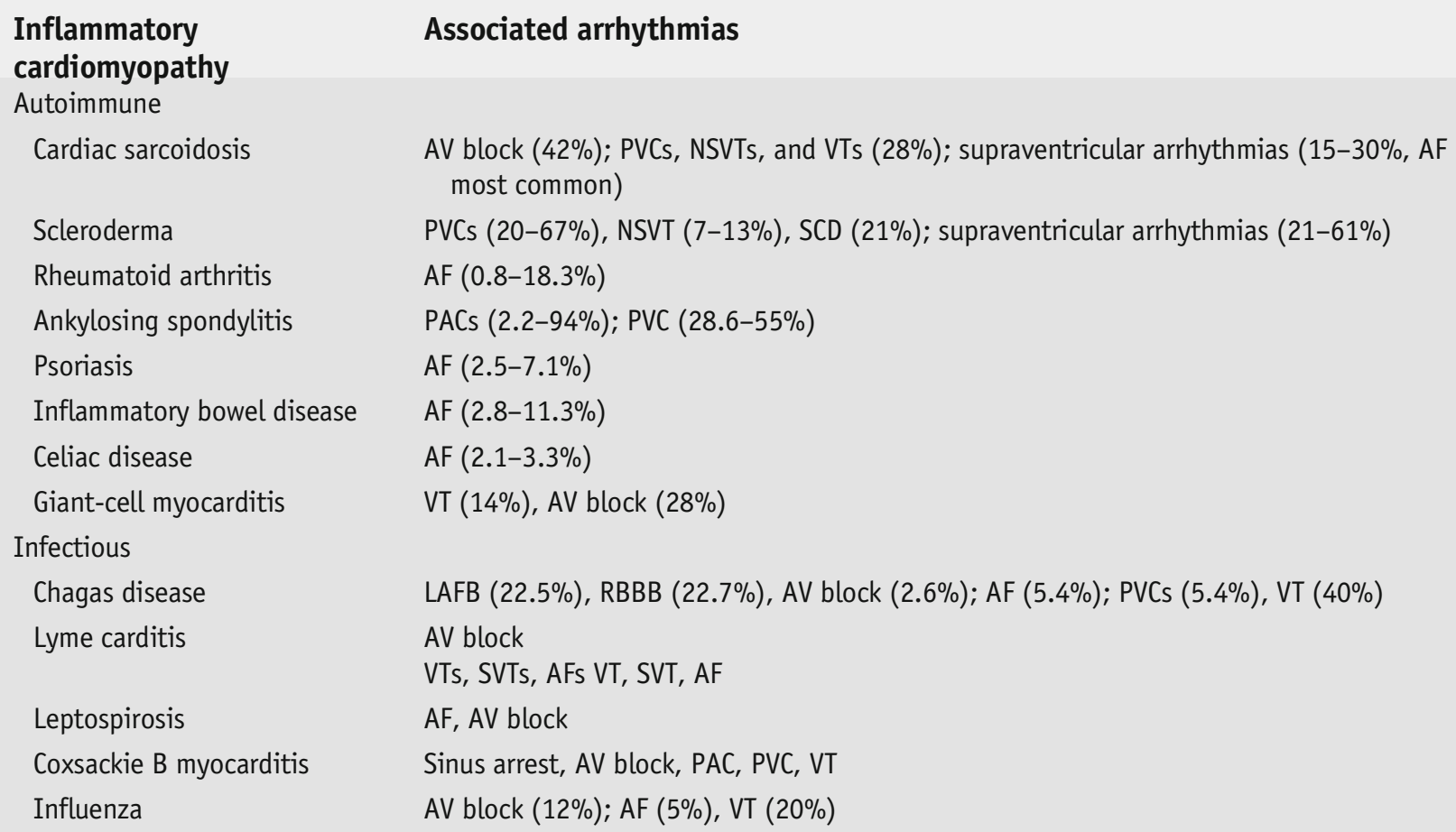


tachycardia (VT) ablation [5]. Clinically manifest CS occurs in 5\% of patients with sarcoidosis. Studies have shown a prevalence of CS to be $6-28 \%$ in patients presenting with nonischemic VTs $[6,7]$, and about $17 \%$ of CS patients present as sudden cardiac arrest [8]. Although giant-cell myocarditis (GCM) is rare, it is an important cause of ICM that can be rapidly fatal. The GCM patients often present with AV block (5-27\%) and ventricular arrhythmias (14-22\%), and about $55 \%$ of the patients develop life-threatening ventricular arrhythmias at the end of 5 years $[9,10]$.

\section{Etiology of inflammatory cardiomyopathy}

Inflammatory cardiomyopathies are caused mostly by (1) infections, (2) autoimmune diseases, and (3) drugs but may be idiopathic. The infectious agents include viruses (coxsackievirus, adenovirus, parvovirus B19, influenza, HIV, hepatitis B, or hepatitis C), bacteria (Borrelia burgdorferi, meningococci, streptococci, psittacosis, diphtheria), rickettsia (typhus, Rocky Mountain spotted fever), fungi (aspergillosis, candidiasis), and parasites (Chagas disease, toxoplasmosis). The Sars-Cov-2 infection responsible for the ongoing COVID-19 pandemic has also been implicated as a cause of acute myocarditis [11]. The rheumatological conditions associated with inflammatory cardiomyopathies include CS, GCM, systemic lupus erythematosus, rheumatoid arthritis, systemic

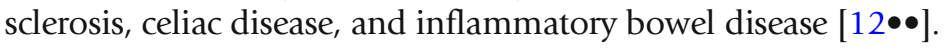

\section{Pathophysiology of arrhythmias in inflammatory cardiomyopathy}

The mechanism of the arrhythmias in ICM depends upon the stages of the inflammation. The primary substrate for the genesis of reentrant ventricular arrhythmias in ICM is tissue heterogeneity resulting from patchy interstitial or replacement fibrosis in the healed phase. However, the slow zones that promote reentry may occur during the stage of active inflammation due to electrical remodeling. In the inflammation-fibrosis continuum, active inflammation results in arrhythmias due to automaticity or triggered activity attributed to various mechanisms, including direct myocardial cell membrane injury, microvascular ischemia (Parvovirus B19), and gap junction dysfunction [13]. Recently, the role of autoimmune channelopathies in the genesis of arrhythmias has also been recognized [14•]. Ongoing active inflammation can also cause polymorphic VT or ventricular fibrillation (VF) akin to acute ischemia. In CS patients, Purkinje-related ventricular arrhythmias have also been described, but their exact mechanisms remain unknown [15]. Interestingly, fibrotic remodeling can also lead to automatic tachycardias by differentiation of fibroblasts into myofibroblasts, which then cause depolarization of electrotonically coupled cardiomyocytes [16]. This process is primarily driven by transforming growth factor- $\beta 1$ (TGF- $\beta 1$ ) [16] and is being explored as a therapeutic target to reduce arrhythmias [17].

Although atrial arrhythmias are more common in CS patients who have late gadolinium enhancement and those with left atrial enlargement, these patients often lack echocardiographic evidence of left ventricular diastolic dysfunction 
or increased left atrial filling pressures [18]. Therefore, direct atrial involvement by granulomatous inflammation and resulting scarring is proposed as a putative mechanism [18]. However, subclinical left ventricular diastolic dysfunction cannot be confidently ruled out, and scarring/inflammation of the atria is challenging to study with currently available imaging technologies. Therefore, the mechanisms of atrial arrhythmias in CS remain to be elucidated.

In ICMs associated with bradycardia, such as CS and GCM myocarditis, there is a predilection for involvement of the conduction system in the basal interventricular septum. The AV block may be due to inflammation, direct mechanical compression (with noncaseating granuloma or giant cells), or fibrosis, or both, resulting in variable response to steroids [19].

\section{Clinical presentation}

Inflammatory cardiomyopathies have a myriad of clinical presentations, from asymptomatic left ventricular systolic dysfunction or mild heart failure to fulminant myocarditis. The patients often present with arrhythmias incident on a previously diagnosed ICM or as the first manifestation. There may or may not be extra-cardiac manifestations at the time of presentation. In addition to symptoms typical of heart failure and arrhythmias, many patients may have subtle symptoms such as fatigue. The common arrhythmic presentations of various ICMs are summarized in Table 1 and discussed briefly in the following paragraphs. The clinical spectrum of CS is summarized in Fig. 1.
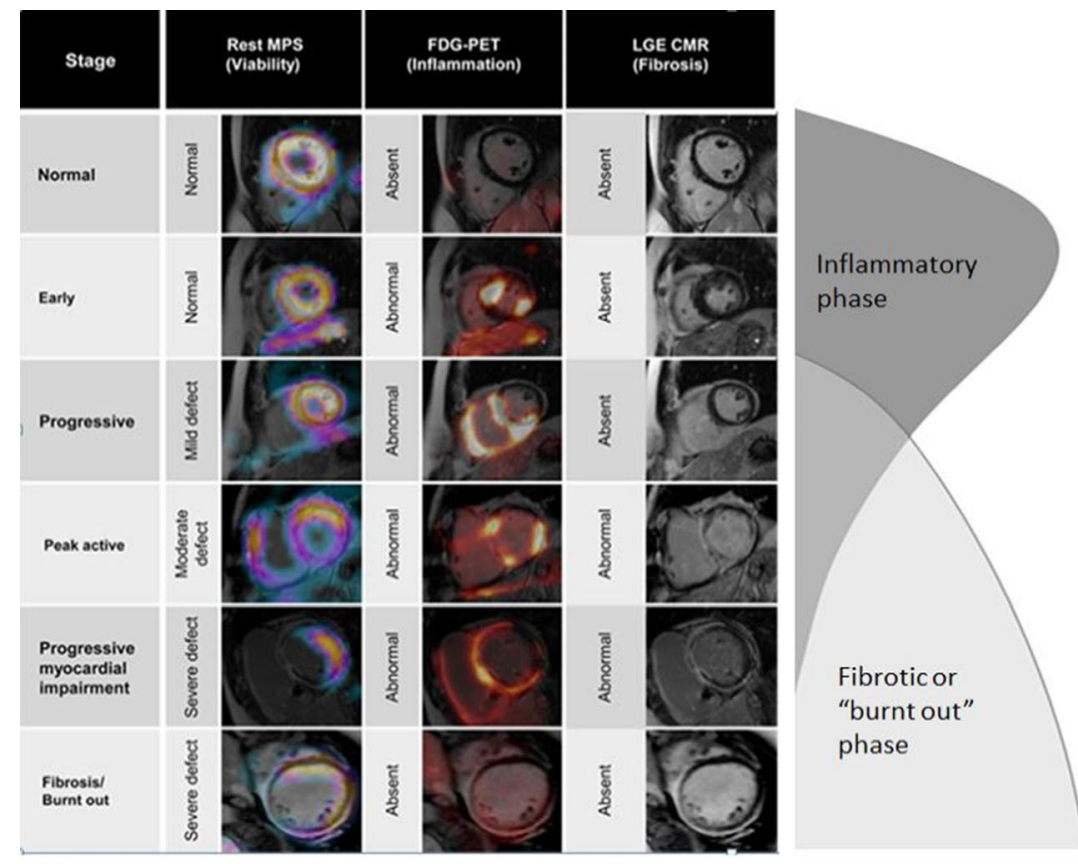

\section{Asymptomatic}

Atrioventricular Block

Direct compression of

conduction system

Ventricular arrhythmias

Automaticity > Re-entry in

inflammatory phase

Reentray >automaticity in

"burnt out" phase

Sudden Cardiac Death

Left ventricular dysfunction

with or without heart failure

Fig. 1. Clinical spectrum of cardiac sarcoidosis from inflammatory to "burnt out" phase with diagnosis based on resting myocardial perfusion SPECT fused with cardiac MRI (left), FDG-PET fused with cardiac MRI (middle), and gadolinium-enhanced cardiac MRI. Adapted with permission from Kouranos et al. [20]. 


\section{Bradyarrhythmias}

For CS, high-grade atrioventricular (AV) block is one of the most common initial presentations in about $42 \%$ of patients [8]. In patients $<60$ years of age presenting with AV block, CS should be suspected $[18,21]$. Other potential differentials include Lyme carditis and giant-cell myocarditis [21, 22]. The CS patients with high-degree AV block are at risk of sudden cardiac death (9-34\% in 5 years) with or without either of left ventricular systolic dysfunction or ventricular arrhythmias [23].

\section{Ventricular arrhythmias and sudden cardiac death}

The patients with acute myocarditis often present with frequent premature ventricular complexes, irregular and polymorphic VT, or ventricular fibrillation [24•]. In contrast, the patients with prior or healed acute myocarditis tend to present with monomorphic and regular ventricular tachycardia [24•]. The patients with CS often have frequent premature ventricular complexes, nonsustained VTs, or sustained VTs. Monomorphic ventricular tachycardia may be an initial presentation of CS with one study showing a prevalence of $28 \%$ in this population [6]. However, the prevalence of CS was reported to be much lower at $5 \%$ in another study $(n=435)$ of patients with nonischemic ventricular tachycardia referred for ablation [7]. The CS patients were noted to have multiple VT circuits (resulting in pleomorphism) and preferential involvement of the interventricular septum and the right ventricle.

Inflammatory cardiomyopathies have been implicated as the cause of sudden cardiac death, especially of young and middle-aged patients $[8,25,26]$. In CS, sudden cardiac arrest may be an initial presentation in about one-seventh of the patients [8]. In an autopsy study of cardiac sarcoidosis patients who had SCD, the predilection for septal or subepicardial involvement was demonstrated [27].

\section{Supraventricular arrhythmias}

Supraventricular arrhythmias are often seen in CS patients (15-32\%) and predominantly include atrial arrhythmias-atrial fibrillation (most common), atrial tachycardia, and atrial flutter-despite their relatively young age $[28,29]$. Interestingly, atrial arrhythmias are more common than the ventricular arrhythmias and often occur in those with left atrial enlargement or presence of late gadolinium enhancement of ventricle(s) on cardiac MRI [28, 29].

\section{Diagnosis}

Focused history to look for an infectious or rheumatic etiology is valuable, but many patients may only have cardiac involvement, making the diagnosis challenging. Family history is paramount as myocarditis may be a red herring for, or arguably feature of, inherited arrhythmogenic cardiomyopathies. In patients with suspected ICM, a constellation of history, imaging studies, and invasive biopsy is required to make a diagnosis. In the 2014 Heart Rhythm Society consensus statement, criteria for the diagnosis of CS (summarized in Table 2) were proposed. 
Table 2. Diagnostic Criteria criteria for Cardiac cardiac Sarcoidosis sarcoidosis Proposed proposed ion the 2014 Heart Rhythm Society Consensus consensus Statementstatement

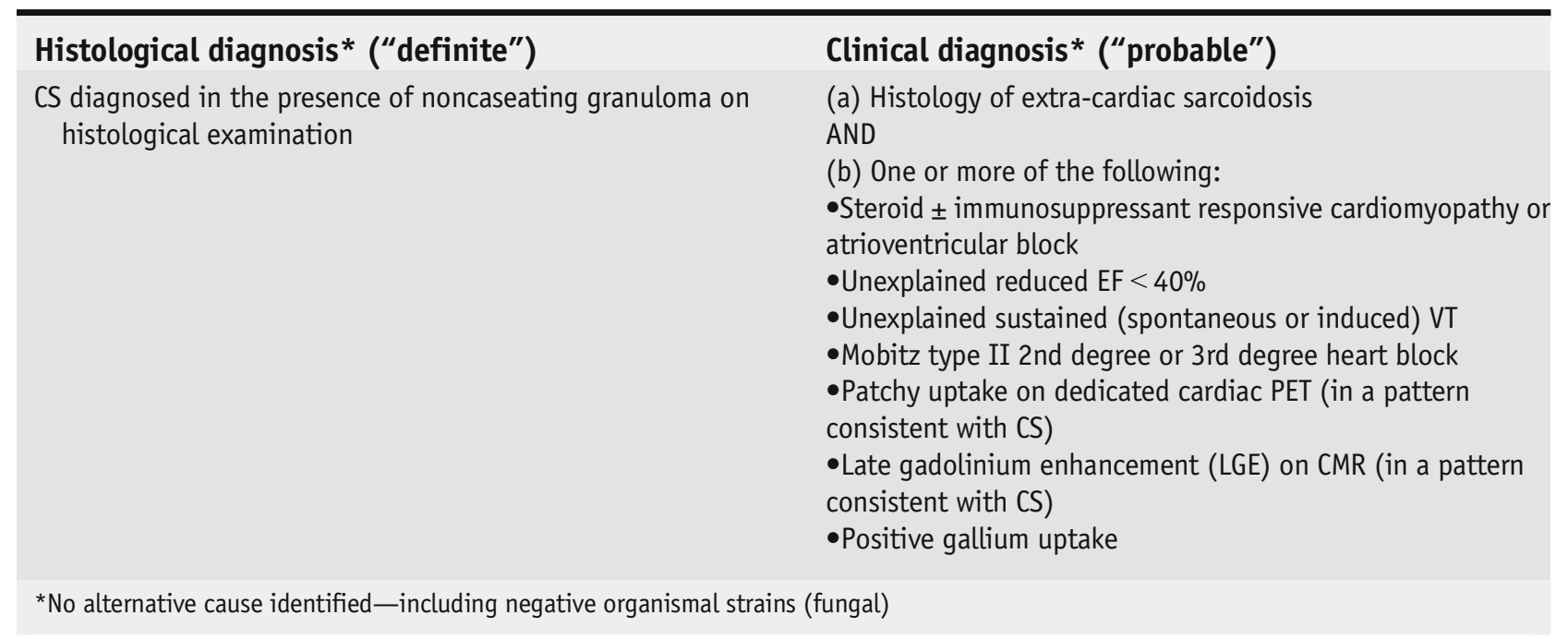

There are serologic tests specific to infections (e.g., Lyme carditis) or inflammatory conditions (e.g., lupus) that can be performed in suspected cases. Acute phase reactants such as sedimentation rate and C-reactive protein may be elevated but are nonspecific. Biomarkers may provide both diagnostic and prognostic information. For example, an elevated B-type natriuretic peptide identifies CS in patients with sarcoidosis with greater accuracy (AUC 0.85) compared to angiotensin-converting enzyme or soluble IL-2, while cardiac troponin I is the strongest predictor of fatal arrhythmias [30].

Echocardiography remains an essential initial study for the assessment of cardiac chamber size and function. The patients tend to have global systolic dysfunction or regional wall motion abnormalities in noncoronary distribution. Although typically characterized by basal septal thinning, some CS patients may have septal hypertrophy in the inflammatory phase of the disease [31].

Late gadolinium enhancement is seen in patients with inflammatory cardiomyopathies, including CS, but the pattern of distribution is nonspecific for diagnosis. CS patients tend to have subepicardial or mid-myocardial involvement. In the early inflammatory phase, late gadolinium enhancement may not be present. The 2009 Lake Louise criteria may be utilized to diagnose myocarditis. However, comprehensive CMR with the mapping of T1 and T2 relaxation times has superior accuracy in acute and chronic myocarditis, respectively [32]. 
In an adequately prepared patient, myocardial uptake of ${ }^{18}$ FDG on PET would indicate the presence of active inflammation. It is important to discuss with the radiologist or imaging cardiologist the sarcoid-specific protocol for preparation of the patients for FDG-PET to avoid false-positive results from physiological uptake (Supplemental Fig. 1). Although FDG-PET likely has utility in most inflammatory cardiomyopathies, it has been extensively studied in CS patients. The focal or focal-on-diffuse pattern would suggest CS as a diagnosis and would also serve as a guide to anti-inflammatory therapy. Therefore, FDG-PET and CMR have complementary roles in the diagnosis of CS patients in different stages (Fig. 1) and their downstream management (Fig. 2) [33]. In addition to cardiac PET, whole-body FDG-PET is useful for the diagnosis of extra-cardiac involvement that could potentially be an easily accessible site for biopsy.

\section{Endomyocardial biopsy}

Because of patchy, preferentially mid-myocardial or subepicardial, and left ventricular involvement, an endomyocardial biopsy has limited sensitivity to justify its routine use for the diagnosis of CS or other inflammatory cardiomyopathies [34]. Therefore, an extra-cardiac biopsy is often performed to support the diagnosis in the presence of other findings suggesting cardiac involvement [35]. The diagnostic yield of the endomyocardial biopsy may be increased by left ventricular or biventricular biopsy [36]. There has been increasing experience with abnormal or low-voltage electrogram-guided biopsy (Fig. 3) to increase the diagnostic yield [37]. According to 2007 ACC/AHA/ESC scientific statement, endomyocardial biopsy is recommended (class I) in patients with

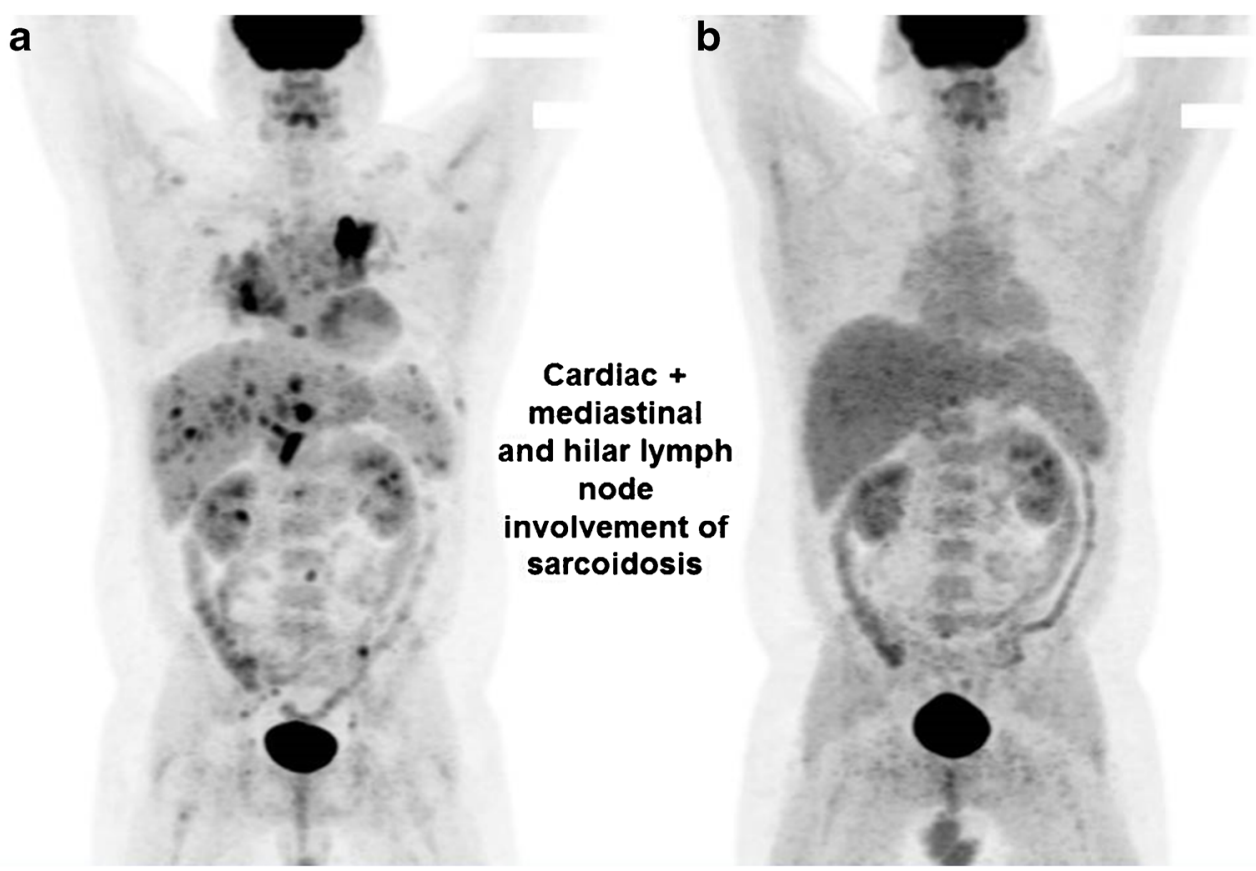

Fig. 2. FDG-PET of patients with cardiac and extra-cardiac sarcoidosis before (a) and after (b) treatment with anti-inflammatory agents (courtesy: Lori A. Blauwet, MD). 

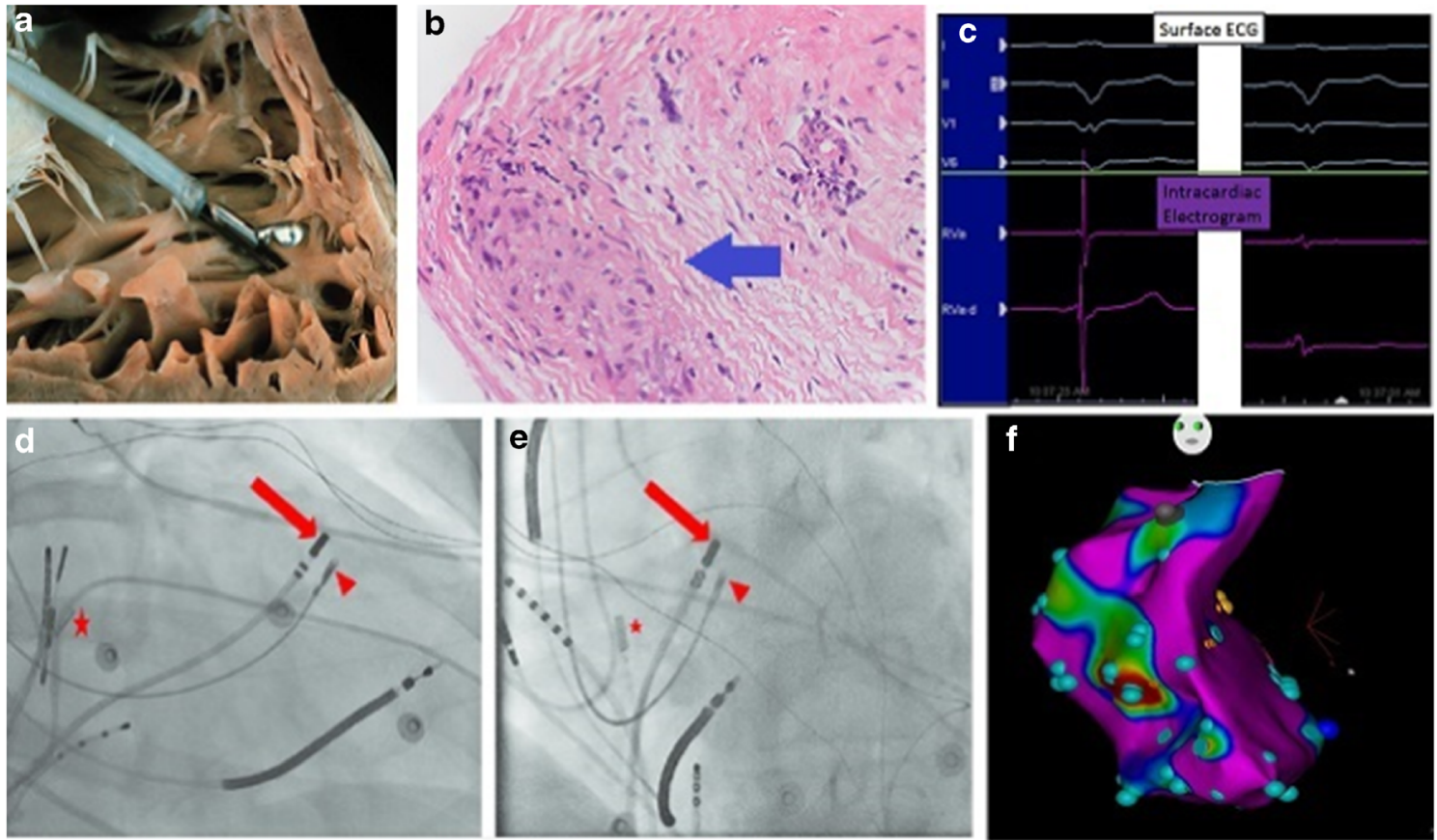

Fig. 3. Endomyocardial biopsy of cardiac sarcoidosis. a Traditional endomyocardial biopsy with a bioptome. b Noncaseating granuloma suggesting a diagnosis of cardiac sarcoidosis. c The abnormal electrogram (right) is lower in voltages and fractionated. d, e RAO and LAO views of EGM-guided biopsy (red arrow, mapping catheter; red triangle, bioptome; star, intracardiac echocardiography). $\mathbf{f}$ Voltage map of patient with sarcoidosis created during biopsy with blue points representing abnormal bipolar signals. d, e Reproduced with permission from Liang et al. [37].

new-onset heart failure ( 2 weeks to 3 months) with new ventricular arrhythmias, 2nd or 3rd degree AV block, or refractory to standard treatment within 12 weeks [38]. The primary aim of this recommendation is to identify giant-cell myocarditis that is rare but can be rapidly fatal without prompt and often advanced therapies.

\section{Management}

The treatment strategy depends upon the patient's clinical presentation and stage of the disease process. The principle of management of CS patients is summarized in Fig. 4 but applies to other ICMs as well.

Medical therapy

All patients who have left ventricular systolic dysfunction with or without clinical heart failure are treated with guideline-directed medical therapy. It is reasonable to consider antiarrhythmic therapy as the first-line management of atrial and ventricular arrhythmias in both the inflammatory and the "burnt out" phases of the disease. The data to guide the choice of an antiarrhythmic drug is limited. While class III drugs such as sotalol and amiodarone are often used initially, concurrent pulmonary involvement should be taken into consideration. 


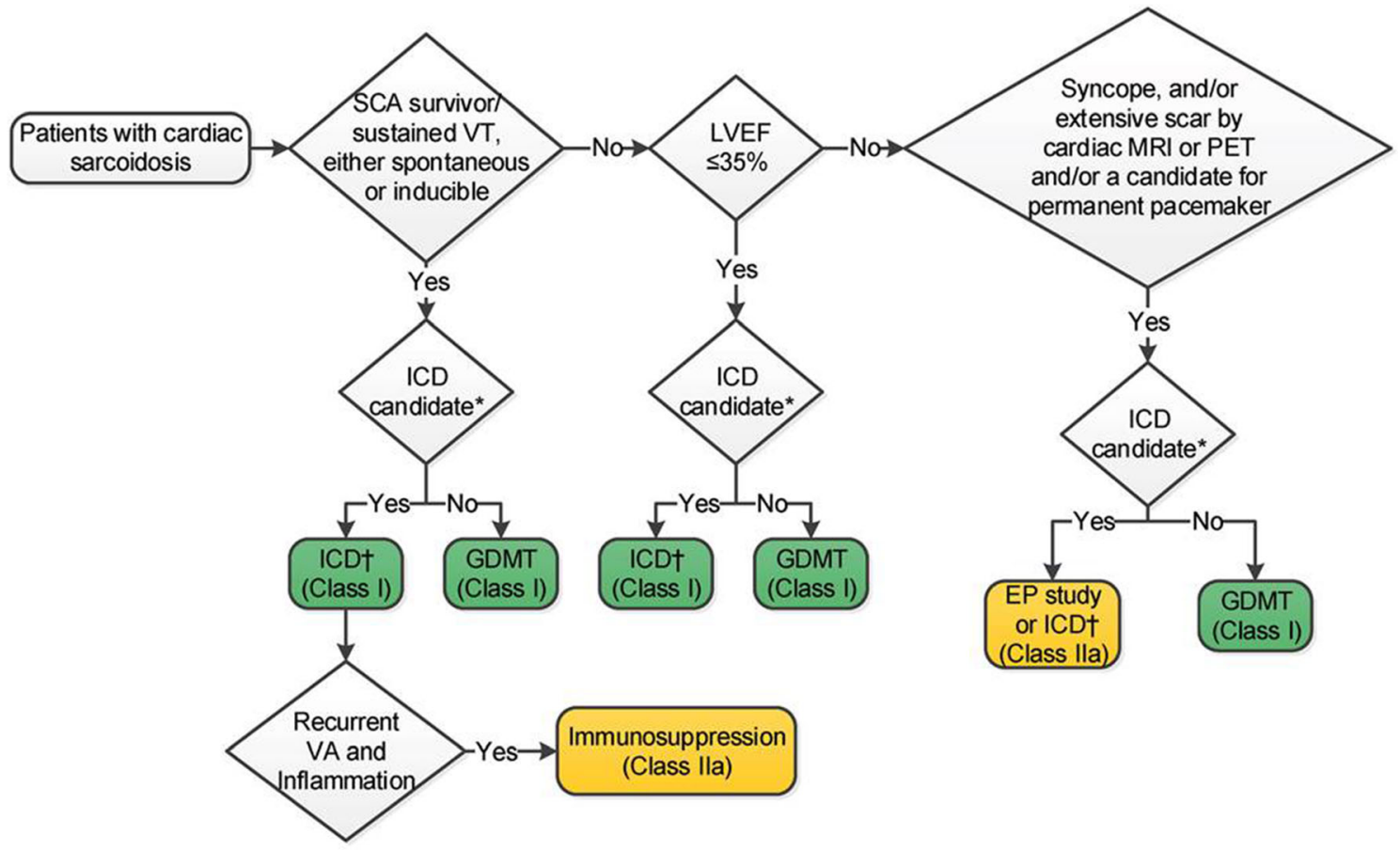

Fig. 4. The 2017 guidelines for ICD for prevention of SCD in patients with cardiac sarcoidosis. Reprinted with permission from AlKhatib et al. [39].

It is intuitive to consider anti-inflammatory therapy in patients who demonstrate active inflammation by FDG-PET; its usefulness for the treatment of arrhythmias remains uncertain. In a meta-analysis by Sadek [40], AV conduction improved in $47 \%$ of patients who received corticosteroid but failed to improve in any patients that did not receive corticosteroid. However, the influence of corticosteroids on the outcome of left ventricular systolic function or ventricular arrhythmias could not be conclusively determined. Some patients treated with corticosteroids may develop electrical storms, mostly within the first year of therapy [41]. Once corticosteroid is started, the CS patients are followed clinically and with FDG-PET at 3-6 months. If there is an improvement of FDG uptake, steroid-sparing agents such as methotrexate should be considered, and corticosteroids are tapered off gradually over the next 69 months. The role of other steroid-sparing agents (e.g., mycophenolate mofetil or azathioprine) is less well-defined, and the utility of biologic agents is unknown.

\section{Device therapy and risk stratification for SCD}

Although SCD remains the most feared outcome of inflammatory cardiomyopathies, the tools to risk-stratify these patients are limited. Similar to other cardiomyopathies, ICD is implanted in CS patients who have sustained ventricular arrhythmias (secondary prevention) or those who have LVEF $\leq 35 \%$ despite optimal medical therapy, including immunosuppressive therapy if FDG positive (primary prevention). In patients with myocarditis, the presence of late gadolinium enhancement on CMR or endocardial bipolar low-voltage areas on 
electro-anatomical mapping has been associated with increased arrhythmic risk [42-44]. Cardiac pacing is indicated in CS patients with high-degree AV block, as it does not improve with corticosteroid therapy in about half of the patients [40]. Irrespective of left ventricular systolic dysfunction or ventricular arrhythmias, these patients are at increased risk of SCD. Programmed ventricular stimulation has been demonstrated to predict arrhythmic events in CS patients $[45,46]$. Therefore, it is also reasonable to implant ICD in the presence of (1) syncope or near-syncope concerning for arrhythmic etiology on CMR, (2) sustained ventricular arrhythmia inducible with programmed electrical stimulation, or (3) indication for cardiac pacing [35]. Given a high risk of SCD in CS patients with mildly reduced ejection fraction (LVEF $>35 \%$ and $<50 \%$ or RVEF $<40 \%$ ), ICD may be considered especially in the presence of LGE or sustained inducible VT on programmed ventricular stimulation (Fig. 3). In the presence of LGE and inducible VT, ICD may be useful in patients with preserved systolic function.

There is limited evidence of the utility of cardiac resynchronization therapy in CS patients. One study $(n=18)$ showed poor reverse remodeling and increased ventricular arrhythmias [47], and a second study $(n=19)$ showed improvement of LVEF, but there was no improvement of LV dimensions, mitral regurgitation, or RV systolic function when compared with other nonischemic cardiomyopathies [48]. Until we have more evidence, it is reasonable to implant a biventricular device or upgrade a previously implanted device in line with the current heart failure guidelines.

Although CS confers a substrate for reentrant VT, catheter ablation can be challenging due to the complexity of substrates with multiple circuits and subepicardial locations likely requiring an epicardial ablation [49-52]. Besides, catheter ablation has been shown to have a lower likelihood for acute success and a higher recurrence rate if performed in the inflammatory phase compared to the healed phase [50•]. Therefore, catheter ablation is reserved for patients with ventricular arrhythmias refractory to immunosuppressive and antiarrhythmic therapies, sometimes early in the course for management of incessant ventricular tachycardia or electrical storm. For CS patients with atrial arrhythmias, catheter ablation has been demonstrated to be feasible with reasonable efficacy and safety [53].

\section{Treatment of refractory ventricular tachycardia}

The patients with ventricular tachycardia refractory to antiarrhythmic therapy and catheter ablation may require cardiac sympathectomy as adjunctive therapy, failing which a mechanical circulatory device such as a left ventricular assist device and cardiac transplantation will need to be considered [7].

\section{Multidisciplinary team approach}

Given the heterogeneity and complexity of inflammatory cardiomyopathies and associated arrhythmias, the key to an effective acute and long-term management is a multidisciplinary team including cardiac electrophysiologists, heart failure cardiologists, imaging cardiologists, pulmonologists, and rheumatologists (Fig. 5). 


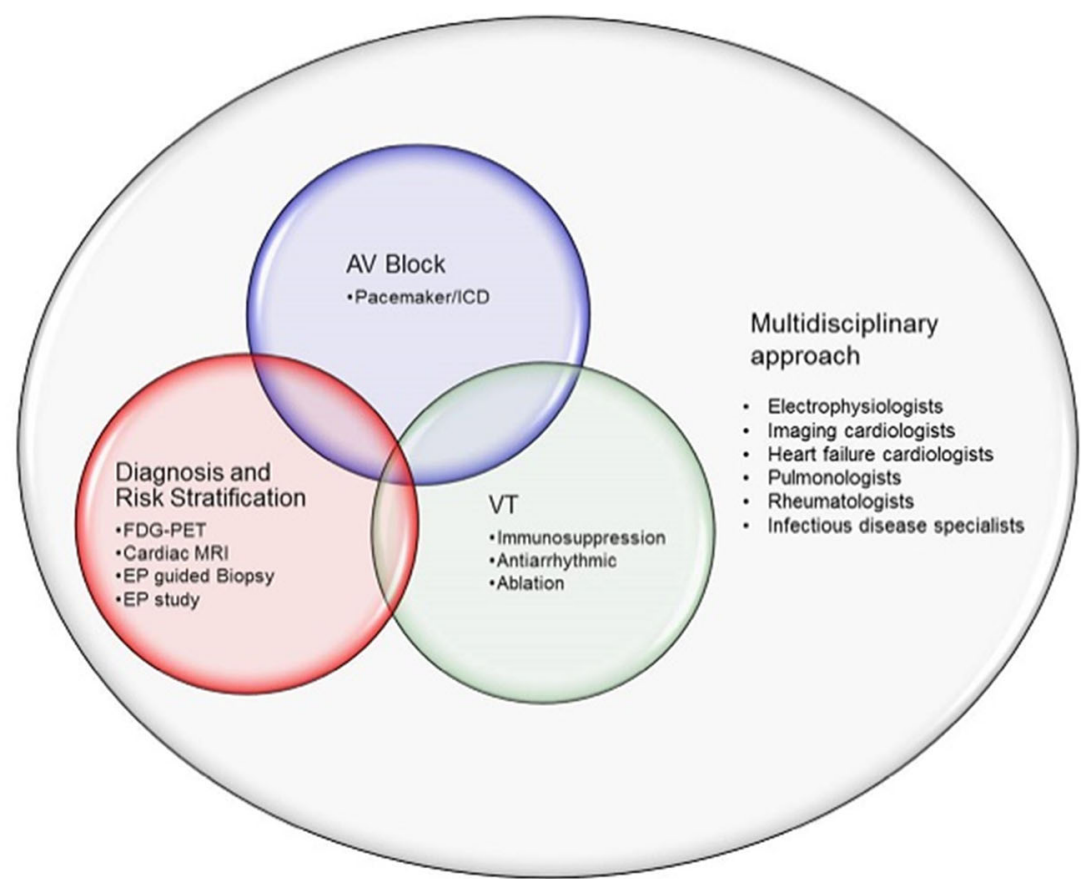

Fig. 5. Principle of arrhythmias associated with cardiac sarcoidosis and other inflammatory cardiomyopathies. Multidisciplinary approach is key to management of these complex diseases.

\section{Future directions}

\section{Conclusions}

Much remains to be known about pathophysiology, diagnosis, and treatment of arrhythmias associated with ICMs, including CS. For the more streamlined treatment of CS patients, there is a need for randomized controlled trials to determine the outcomes of immune suppression, antiarrhythmics, catheter ablation, and device therapy. The Cardiac Sarcoidosis Multi-Center Randomized Controlled Trial (CHASM CS-RCT, NCT03593759) is seeking to determine the noninferiority in the efficacy of low-dose prednisone and methotrexate when compared with standard-dose prednisone, in addition to the superiority in quality of life measures [54]. The Interleukin-1 Blockade for Treatment of Cardiac Sarcoidosis Trial (MAGiC-ART, NCT04017936) will study the effect of anakinra on cardiac inflammation and fibrosis.

Inflammatory cardiomyopathies often have an arrhythmic presentation, with AV block, atrial arrhythmias, or ventricular arrhythmias, including SCD. Given their heterogeneous nature, accurate diagnosis and risk stratification can be challenging. A multidisciplinary approach is important to guide effective treatment. 


\section{Supplementary Information}

The online version of this article (https://doi.org/10.1007/s11936-020-00871-5) contains supplementary material, which is available to authorized users.

\section{Compliance with Ethical Standards}

\section{Conflict of interest}

The authors has no conflicts of interest to declare.

Animal rights and informed consent statement

This article does not contain any studies with human or animal subjects performed by any of the authors.

Code availability

Not applicable

\section{References and Recommended Reading}

Papers of particular interest, published recently, have been highlighted as:

- Of importance

- Of major importance

1. Deshmukh A, Sewani A, Sachdeva R. Cardiac sarcoidosis masquerading as syncope with right ventricular septal mass. J Invasive Cardiol. 2012;24(8):418-9.

2.• Bauer BS, Li A, Bradfield JS. Arrhythmogenic inflammatory cardiomyopathy: a review. Arrhythmia Electrophysiol Rev. 2018;7(3):181-6. https://doi.org/ 10.15420/aer.2018.26.2

This paper tries to define inflammatory cardiomyopathies causing arrhythmias as a distinct entity called arrhythmogenic inflammatory cardiomyopathy.

3. James SL, Abate D, Abate KH, Abay SM, Abbafati C, Abbasi N, et al. Global, regional, and national incidence, prevalence, and years lived with disability for 354 diseases and injuries for 195 countries and territories, 1990-2017: a systematic analysis for the Global Burden of Disease Study 2017. Lancet. 2018;392(10159):1789-858. https://doi.org/10. 1016/S0140-6736(18)32279-7.

4. Lynge TH, Nielsen TS, Gregers Winkel B, Tfelt-Hansen J, Banner J. Sudden cardiac death caused by myocarditis in persons aged 1-49 years: a nationwide study of 14294 deaths in Denmark. Forensic Sciences Research. 2019;4(3):247-56. https://doi.org/10.1080/ 20961790.2019.1595352.
5. Tung R, Bauer B, Schelbert H, Lynch JP 3rd, Auerbach M, Gupta P, et al. Incidence of abnormal positron emission tomography in patients with unexplained cardiomyopathy and ventricular arrhythmias: the potential role of occult inflammation in arrhythmogenesis. Heart Rhythm. 2015;12(12):248898. https://doi.org/10.1016/j.hrthm.2015.08.014.

6. Nery PB, Mc Ardle BA, Redpath CJ, Leung E, Lemery R, Dekemp R, et al. Prevalence of cardiac sarcoidosis in patients presenting with monomorphic ventricular tachycardia. PACE - Pacing Clin Electrophysiol. 2014;37(3):364-74. https://doi.org/10.1111/pace. 12277.

7. Koplan BA, Soejima K, Baughman K, Epstein LM, Stevenson WG. Refractory ventricular tachycardia secondary to cardiac sarcoid: electrophysiologic characteristics, mapping, and ablation. Heart Rhythm. 2006;3(8):924-9. https://doi.org/10.1016/j.hrthm. 2006.03.031.

8. Ekström K, Lehtonen J, Nordenswan H-K, Mäyränpää MI, Räisänen-Sokolowski A, Kandolin R, et al. Sudden death in cardiac sarcoidosis: an analysis of nationwide clinical and cause-of-death registries. Eur Heart J. 
2019;40(37):3121-8. https://doi.org/10.1093/ eurheartj/ehz428.

9. Ekström K, Lehtonen J, Kandolin R, RäisänenSokolowski A, Salmenkivi K, Kupari M. Incidence, risk factors, and outcome of life-threatening ventricular arrhythmias in giant cell myocarditis. Circ Arrhythm Electrophysiol. 2016;9(12):e004559. https://doi.org/ 10.1161/CIRCEP.116.004559.

10. Cooper LT, Berry GJ, Shabetai R. Idiopathic giant-cell myocarditis - natural history and treatment. N Engl J Med. 1997;336(26):1860-6. https://doi.org/10.1056/ nejm199706263362603.

11. Dhakal BP, Sweitzer NK, Indik JH, Acharya D, William P. SARS-CoV-2 infection and cardiovascular disease: COVID-19 heart. Heart Lung Circ. 2020. https://doi. org/10.1016/j.hlc.2020.05.101.

12.• Mavrogeni SI, Markousis-Mavrogenis G, Aggeli C, Tousoulis D, Kitas GD, Kolovou G, et al. Arrhythmogenic inflammatory cardiomyopathy in autoimmune rheumatic diseases: a challenge for cardio-rheumatology. Diagnostics (Basel). 2019;9(4):217. https://doi. org/10.3390/diagnostics9040217

This paper is the most recent and comprehensive review of arrhythmogenic inflammatory cardiomyopathies caused by autoimmune diseases.

13. Peretto G, Sala S, Rizzo S, De Luca G, Campochiaro C, Sartorelli S, et al. Arrhythmias in myocarditis: state of the art. Heart Rhythm. 2019;16(5):793-801. https:// doi.org/10.1016/j.hrthm.2018.11.024.

14. Lazzerini PE, Capecchi PL, El-Sherif N, Laghi-Pasini F, Boutjdir M. Emerging arrhythmic risk of autoimmune and inflammatory cardiac channelopathies. J Am Heart Assoc. 2018;7(22):e010595. https://doi.org/10.1161/ JAHA.118.010595

This paper summarizes the non-inherited cardiac channelopathies that may be responsible for genesis of arrhythmias associated with inflammatory cardiomyopathy.

15. Naruse Y, Sekiguchi Y, Nogami A, Okada H, Yamauchi Y, MacHino T, et al. Systematic treatment approach to ventricular tachycardia in cardiac sarcoidosis. Circ Arrhythm Electrophysiol. 2014;7(3):407-13. https:// doi.org/10.1161/CIRCEP.113.000734.

16. Salvarani N, Maguy A, De Simone SA, Miragoli M, Jousset F, Rohr S. TGF- $\beta(1)$ (transforming growth factor- $\beta(1)$ ) plays a pivotal role in cardiac myofibroblast arrhythmogenicity. Circ Arrhythm Electrophysiol. 2017;10(5):e004567. https://doi.org/10.1161/circep. 116.004567

17. Nguyen DT, Ding C, Wilson E, Marcus GM, Olgin JE. Pirfenidone mitigates left ventricular fibrosis and dysfunction after myocardial infarction and reduces arrhythmias. Heart Rhythm. 2010;7(10):1438-45. https://doi.org/10.1016/j.hrthm.2010.04.030.

18. Nery PB, Beanlands RS, Nair GM, Green M, Yang J, McArdle BA, et al. Atrioventricular block as the initial manifestation of cardiac sarcoidosis in middle-aged adults. J Cardiovasc Electrophysiol. 2014;25(8):87581. https://doi.org/10.1111/jce.12401.
19. Orii M, Hirata K, Tanimoto T, Ota S, Shiono Y, Yamano $\mathrm{T}$, et al. Comparison of cardiac MRI and 18F-FDG positron emission tomography manifestations and regional response to corticosteroid therapy in newly diagnosed cardiac sarcoidosis with complete heart block. Heart Rhythm. 2015;12(12):2477-85. https://doi.org/ 10.1016/j.hrthm.2015.06.032.

20. Kouranos et al. British Medical Bulletin 2015, 115, 1, 156 (publisher: Oxford University Press).

21. Kandolin R, Lehtonen J, Kupari M. Cardiac sarcoidosis and giant cell myocarditis as causes of atrioventricular block in young and middle-aged adults. Circ Arrhythm Electrophysiol. 2011;4(3):303-9. https://doi.org/10. 1161/CIRCEP.110.959254.

22. Yeung C, Baranchuk A. Diagnosis and treatment of Lyme carditis: JACC review topic of the week. J Am Coll Cardiol. 2019;73(6):717-26. https://doi.org/10.1016/ j.jacc.2018.11.035.

23. Nordenswan HK, Lehtonen J, Ekström K, Kandolin R, Simonen P, Mäyränpää M, et al. Outcome of cardiac sarcoidosis presenting with high-grade atrioventricular block. Circ Arrhythm Electrophysiol.

2018;11(8):e006145. https://doi.org/10.1161/ CIRCEP.117.006145.

24. Peretto G, Sala S, Rizzo S, Palmisano A, Esposito A, De Cobelli $\mathrm{F}$, et al. Ventricular arrhythmias in myocarditis: characterization and relationships with myocardial inflammation. J Am Coll Cardiol. 2020;75(9):1046-57. https://doi.org/10.1016/j.jacc.2020.01.036

This paper is a recent review on this topic but also highlights possible pathways for arrhythmogenesis in inflammatory cardiomyopathies and these could be a potential target for drug development in future.

25. Fabre A, Sheppard MN. Sudden adult death syndrome and other non-ischaemic causes of sudden cardiac death. 2006. p. 316-20.

26. Eckart RE, Scoville SL, Campbell CL, Shry EA, Stajduhar KC, Potter RN et al. Sudden death in young adults: a 25 -year review of autopsies in military recruits. American College of Physicians; 2004. p. 829-34.

27. Tavora F, Cresswell N, Li L, Ripple M, Solomon C, Burke A. Comparison of necropsy findings in patients with sarcoidosis dying suddenly from cardiac sarcoidosis versus dying suddenly from other causes. Am J Cardiol. 2009;104(4):571-7. https://doi.org/10.1016/ j.amjcard.2009.03.068.

28. Viles-Gonzalez JF, Pastori L, Fischer A, Wisnivesky JP, Goldman MG, Mehta D. Supraventricular arrhythmias in patients with cardiac sarcoidosis: prevalence, predictors, and clinical implications. Chest. 2013;143(4):1085-90. https://doi.org/10.1378/chest. 11-3214.

29. Cain MA, Metzl MD, Patel AR, Addetia K, Spencer KT, Sweiss NJ, et al. Cardiac sarcoidosis detected by late gadolinium enhancement and prevalence of atrial arrhythmias. Am J Cardiol. 2014;113(9):1556-60. https://doi.org/10.1016/j.amjcard.2014.01.434.

30. Kiko T, Yoshihisa A, Kanno Y, Yokokawa T, Abe S, Miyata-Tatsumi M, et al. A multiple biomarker 
approach in patients with cardiac sarcoidosis. Int Heart J. 2018;59(5):996-1001. https://doi.org/10.1536/ihj. 17-695.

31. Agarwal A, Sulemanjee NZ, Cheema O, Downey FX, Tajik AJ. Cardiac sarcoid: a chameleon masquerading as hypertrophic cardiomyopathy and dilated cardiomyopathy in the same patient. Echocardiography. 2014;31(5):E138-41. https://doi.org/10.1111/echo. 12536.

32. Ferreira VM, Schulz-Menger J, Holmvang G, Kramer $\mathrm{CM}$, Carbone I, Sechtem U, et al. Cardiovascular magnetic resonance in nonischemic myocardial inflammation. Expert Recomm. 2018;72(24):3158-76. https://doi.org/10.1016/j.jacc.2018.09.072.

33. Vita T, Okada DR, Veillet-Chowdhury M, Bravo PE, Mullins E, Hulten E, et al. Complementary value of cardiac magnetic resonance imaging and positron emission tomography/computed tomography in the assessment of cardiac sarcoidosis. Circ Cardiovasc Imaging. 2018;11(1):e007030. https://doi.org/10.1161/ CIRCIMAGING.117.007030.

34. Uemura A, Morimoto S, Hiramitsu S, Kato Y, Ito T, Hishida H. Histologic diagnostic rate of cardiac sarcoidosis: evaluation of endomyocardial biopsies. Am Heart J. 1999;138(2 Pt 1):299-302. https://doi.org/10. 1016/s0002-8703(99)70115-8.

35. Birnie DH, Sauer WH, Bogun F, Cooper JM, Culver DA, Duvernoy CS, et al. HRS expert consensus statement on the diagnosis and management of arrhythmias associated with cardiac sarcoidosis. Heart Rhythm. 2014;11(7):1305-23. https://doi.org/10.1016/j. hrthm.2014.03.043.

36. Chimenti C, Frustaci A. Contribution and risks of left ventricular endomyocardial biopsy in patients with cardiomyopathies. Circulation. 2013;128(14):153141. https://doi.org/10.1161/CIRCULATIONAHA.13. 001414.

37. Liang JJ, Hebl VB, DeSimone CV, Madhavan M, Nanda S, Kapa S, et al. Electrogram guidance: a method to increase the precision and diagnostic yield of endomyocardial biopsy for suspected cardiac sarcoidosis and myocarditis. JACC Heart Fail. 2014;2(5):46673. https://doi.org/10.1016/j.jchf.2014.03.015.

38. Cooper LT, Baughman KL, Feldman AM, Frustaci A, Jessup M, Kuhl U, et al. The role of endomyocardial biopsy in the management of cardiovascular disease: a scientific statement from the American Heart Association, the American College of Cardiology, and the European Society of Cardiology. Endorsed by the Heart Failure Society of America and the Heart Failure Association of the European Society of Cardiology. J Am Coll Cardiol. 2007;50(19):1914-31. https://doi.org/ 10.1016/j.jacc.2007.09.008.

39. Al-Khatib et al. Circulation, 2018, 138, 13 (publisher: Wolters Kluwer).

40. Sadek MM, Yung D, Birnie DH, Beanlands RS, Nery PB. Corticosteroid therapy for cardiac sarcoidosis: a systematic review. 2013. p. 1034-41.
41. Segawa M, Fukuda K, Nakano M, Kondo M, Satake H, Hirano $\mathrm{M}$, et al. Time course and factors correlating with ventricular tachyarrhythmias after introduction of steroid therapy in cardiac sarcoidosis. Circ Arrhythm Electrophysiol. 2016;9(6):e003353. https://doi.org/ 10.1161/CIRCEP.115.003353.

42. Aquaro GD, Perfetti M, Camastra G, Monti L, Dellegrottaglie S, Moro C, et al. Cardiac MR with late gadolinium enhancement in acute myocarditis with preserved systolic function: ITAMY study. J Am Coll Cardiol. 2017;70(16):1977-87. https://doi.org/10. 1016/j.jacc.2017.08.044.

43. Grani C, Eichhorn C, Biere L, Murthy VL, Agarwal V, Kaneko K, et al. Prognostic value of cardiac magnetic resonance tissue characterization in risk stratifying patients with suspected myocarditis. J Am Coll Cardiol. 2017;70(16):1964-76. https://doi.org/10.1016/j.jacc. 2017.08.050.

44. Pelargonio G, Pinnacchio G, Narducci ML, Pieroni M, Perna F, Bencardino G, et al. Long-term arrhythmic risk assessment in biopsy-proven myocarditis. JACC Clin Electrophysiol. 2020;6(5):574-82. https://doi.org/10. 1016/j.jacep.2019.12.010

This paper describes the difference in ventricular arrhythmias based on the stages of inflammatory cardiomyopathy. 45. Mehta D, Mori N, Goldbarg SH, Lubitz S, Wisnivesky JP, Teirstein A. Primary prevention of sudden cardiac death in silent cardiac sarcoidosis role of programmed ventricular stimulation. Circ Arrhythm Electrophysiol. 2011;4(1):43-8. https://doi.org/10.1161/CIRCEP. 110.958322 .

46. Aizer A, Stern EH, Gomes JA, Teirstein AS, Eckart RE, Mehta D. Usefulness of programmed ventricular stimulation in predicting future arrhythmic events in patients with cardiac sarcoidosis. Am J Cardiol. 2005;96(2):276-82. https://doi.org/10.1016/j. amjcard.2005.03.059.

47. Sairaku A, Yoshida Y, Nakano Y, Hirayama H, Maeda $\mathrm{M}$, Hashimoto $\mathrm{H}$, et al. Cardiac resynchronization therapy for patients with cardiac sarcoidosis. EP Europace. 2017;19(5):824-30. https://doi.org/10. 1093/europace/euw223.

48. Patel D, Trulock KM, Toro S, Grimaldi A, Gonzalez M, Moennich LA, et al. Effect of cardiac resynchronization therapy on left ventricular remodeling in patients with cardiac sarcoidosis. Am J Cardiol. 2019;123(2):32933. https://doi.org/10.1016/j.amjcard.2018.09.044.

49. Kumar S, Barbhaiya C, Nagashima K, Choi E-K, Epstein LM, John RM, et al. Ventricular tachycardia in cardiac sarcoidosis. Circ Arrhythm Electrophysiol. 2015;8(1):87-93. https://doi.org/10.1161/CIRCEP. 114.002145 .

50. Kaur D, Roukoz H, Shah M, Yalagudri S, Pandurangi U, Chennapragada $S$, et al. Impact of the inflammation on the outcomes of catheter ablation of drug-refractory ventricular tachycardia in cardiac sarcoidosis. J Cardiovasc Electrophysiol. 2020;31(3):612-20. https://doi.org/10.1111/jce.14341

This is an important paper looking at the role of catheter 
ablation in the management of cardiac sarcoidosis depending upon the phases of inflammation.

51. Muser D, Santangeli P, Pathak RK, Castro SA, Liang JJ, Magnani S, et al. Long-term outcomes of catheter ablation of ventricular tachycardia in patients with cardiac sarcoidosis. Circ Arrhythm Electrophysiol. 2016;9(8):e004333. https://doi.org/10.1161/CIRCEP. 116.004333.

52. Papageorgiou N, Providência R, Bronis K, Dechering DG, Srinivasan N, Eckardt L, et al. Catheter ablation for ventricular tachycardia in patients with cardiac sarcoidosis: a systematic review. Europace. 2018;20(4):682-91. https://doi.org/10.1093/ europace/eux077.

53. Willner JM, Viles-Gonzalez JF, Coffey JO, Morgenthau AS, Mehta D. Catheter ablation of atrial arrhythmias in cardiac sarcoidosis. J Cardiovasc Electrophysiol.
2014;25(9):958-63. https://doi.org/10.1111/jce. 12424.

54. Birnie D, Beanlands RSB, Nery P, Aaron SD, Culver DA, DeKemp RA, et al. Cardiac sarcoidosis multi-center randomized controlled trial (CHASM CS- RCT). Am Heart J. 2020;220:246-52. https://doi.org/10.1016/j. ahj.2019.10.003

\section{Publisher's Note}

Springer Nature remains neutral with regard to jurisdictional claims in published maps and institutional affiliations. 